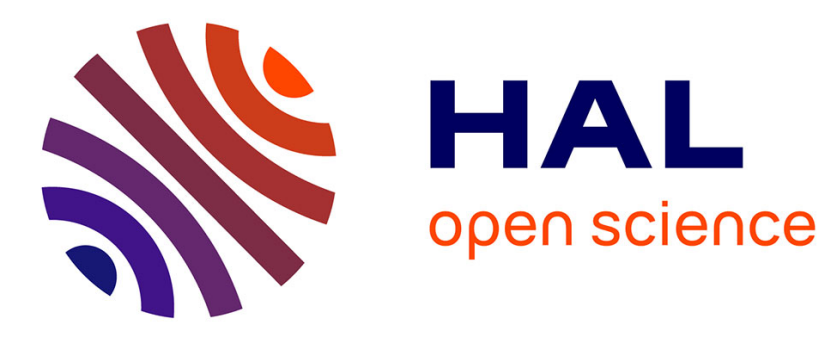

\title{
Two-dimensional inverse profiling problem using phaseless data
}

Amelie Litman, Kamal Belkebir

\section{To cite this version:}

Amelie Litman, Kamal Belkebir. Two-dimensional inverse profiling problem using phaseless data. Journal of the Optical Society of America. A Optics, Image Science, and Vision, 2006, 23 (11), pp.2737 - 2746. 10.1364/JOSAA.23.002737 . hal-00082943

\section{HAL Id: hal-00082943 \\ https://hal.science/hal-00082943}

Submitted on 12 Nov 2018

HAL is a multi-disciplinary open access archive for the deposit and dissemination of scientific research documents, whether they are published or not. The documents may come from teaching and research institutions in France or abroad, or from public or private research centers.
L'archive ouverte pluridisciplinaire HAL, est destinée au dépôt et à la diffusion de documents scientifiques de niveau recherche, publiés ou non, émanant des établissements d'enseignement et de recherche français ou étrangers, des laboratoires publics ou privés. 


\title{
Two-dimensional inverse profiling problem using phaseless data
}

\author{
Amélie Litman and Kamal Belkebir \\ Institut Fresnel, UMR-CNRS 6133, \\ Campus de Saint Jérôme, case 162 \\ Université de Provence, 13397 Marseille Cedex, France
}

This paper deals with the characterization of two-dimensional targets from their diffracted intensity. The target characterization is performed by minimizing an adequate cost functional, combined with a level-set representation if the target is homogeneous. One key issue in this minimization is the choice of an updating direction, which involves the gradient of the cost functional. This gradient can be evaluated using a fictitious field, solution of an adjoint problem where receivers act as sources with a specific amplitude. We explore the Born approximation for the adjoint field and compare various approaches for a wide variety of objects.

(c) 2007 Optical Society of America

OCIS codes: 290.3200, 110.6960 


\section{Introduction}

In some practical applications, the phase measurement of the scattered fields are too corrupted by noise to be useful or even there is no phase measurement at all, e.g., optical measurement setup. Even if there is some effort nowadays to provide experimental setups which measure all components of the scattered fields ${ }^{1,2}$, our purpose herein is to investigate a method that image samples from the modulus of the scattered field only. Indeed, it has been shown that the scattered intensity could provide useful information on the obstacles. ${ }^{3}$

Instead of extracting some phase information from measurements ${ }^{4}$, and then solving the inverse scattering problem from the measured intensity and the preliminary retrieved phase, we directly retrieve the targets under test from the scattered intensity. Following the ideas of Refs. 5,6, the approach, suggested herein, builds up the parameter of interest, namely the contrast of permittivity, iteratively. It is gradually adjusted by minimizing a cost functional properly defined.

This minimization under constraints is reformulated in terms of a Lagrangian functional, whose saddle point leads to the definition of an adjoint problem. ${ }^{7}$ By virtue of the reciprocity principle, this adjoint problem is equivalent to a forward scattering problem where receivers act as sources with a correctly defined amplitude. It will be shown that the only difference between a standard minimization process using modulus-phase data and this algorithm is expressed in these weighting coefficients. This implies that passing from full data to amplitude data requires only one line change in a software program if an adjoint field formalism is used.

This approach is then introduced for two cases of permittivity profile: a continuous profile 
and a step profile. The first case is solved thanks to a conjugate-gradient type algorithm. For the second case, a level-set representation is introduced, which fully takes into account prior information stating that the obstacle is homogeneous. ${ }^{8}$ Results using modulus only measurements will then be analyzed in a free space configuration for those two cases of permittivity profile. In particular, we highlight with various numerical examples the effect on the gradient computation and on the convergence, of physical approximations such as Born approximation for both the forward and adjoint fields. We also introduce a new initial guess based on an appropriate use of topological derivative, which is no more than the variation of the cost functional due to the inclusions of small dielectric balls. ${ }^{9}$

The following paper is organized as follows. In the first section, a description of the geometry is provided. The second section is devoted to the definition of the inverse scattering problem, with the introduction of the cost functional and the associated Lagrangian formulation. The gradient expression is then provided and several choices of computation are discussed. The third section focuses on the application of this gradient computation to the case of heterogeneous obstacles by means of conjugate-gradient algorithm or to the case of homogeneous obstacles by means of level-sets. The way the initial guess is obtained is also explained in the same section. Finally, the last section provides numerical examples for both homogeneous and heterogeneous obstacles, with and without noise, showing the effects of a correct gradient computation as well as the appropriate use of an a priori information on the nature of the scatterers. 


\section{Statement of the problem}

The geometry of the problem studied in this paper is shown in Fig. (1) where a twodimensional object of arbitrary cross-section $\Omega$ is confined in a bounded domain $D$. The embedding medium $\Omega_{b}$ is assumed to be infinite and homogeneous, with permittivity $\varepsilon_{b}=\varepsilon_{0} \varepsilon_{b r}$, and of permeability $\mu=\mu_{0}\left(\varepsilon_{0}\right.$ and $\mu_{0}$ being the permittivity and permeability of the vacuum, respectively). The scatterers are assumed to be inhomogeneous cylinders with a permittivity distribution $\varepsilon(\mathbf{r})=\varepsilon_{0} \varepsilon_{r}(\mathbf{r})$; the entire configuration is non-magnetic $\left(\mu=\mu_{0}\right)$. A right-handed Cartesian coordinate frame $\left(O, \mathbf{u}_{\mathbf{x}}, \mathbf{u}_{\mathbf{y}}, \mathbf{u}_{\mathbf{z}}\right)$ is defined. The origin $O$ can be either inside or outside the scatterer and the $z$-axis is parallel to the invariance axis of the scatterer. The position vector $\mathbf{O M}$ can then be written as $\mathbf{O M}=\mathbf{r}+z \mathbf{u}_{\mathbf{z}}$. The sources that generate the electromagnetic excitation are assumed to be lines parallel to the $z$-axis, located at $\left(\mathbf{r}_{l}\right)_{1 \leq l \leq L}$. Taking into account a time factor $\exp (-i \omega t)$, in the Transverse Magnetic (TM) case, the time-harmonic incident electric field created by the $l^{\text {th }}$ line source is given by

$$
\mathbf{E}_{l}^{\mathrm{i}}(\mathbf{r})=E_{l}^{\mathrm{i}}(\mathbf{r}) \mathbf{u}_{\mathbf{z}}=P \frac{\omega \mu_{0}}{4} H_{0}^{(1)}\left(k_{b}\left|\mathbf{r}-\mathbf{r}_{l}\right|\right) \mathbf{u}_{\mathbf{z}}
$$

where $P$ is the strength of the electric source, $\omega$ the angular frequency, $H_{0}^{(1)}$ the Hankel function of zero order and of the first kind and $k_{b}$ the wavenumber in the surrounding medium.

For the inverse scattering problem, we assume that the unknown object is successively illuminated by $L$ electromagnetic excitations and for each the scattered field is available along a contour $\Gamma$ at $M$ positions. The direct scattering problem may be formulated as two coupled contrast-source integral relations: the observation equation (Eq. 2) and the coupling 
equation (Eq. 3)

$$
\begin{aligned}
& E_{l}^{\mathrm{s}}(\mathbf{r} \in \Gamma)=k_{0}^{2} \int_{D} \chi\left(\mathbf{r}^{\prime}\right) E_{l}\left(\mathbf{r}^{\prime}\right) G\left(\mathbf{r}, \mathbf{r}^{\prime}\right) \mathrm{d} \mathbf{r}^{\prime}, \\
& E_{l}(\mathbf{r} \in D)=E_{l}^{\mathrm{i}}+k_{0}^{2} \int_{D} \chi\left(\mathbf{r}^{\prime}\right) E_{l}\left(\mathbf{r}^{\prime}\right) G\left(\mathbf{r}, \mathbf{r}^{\prime}\right) \mathrm{d} \mathbf{r}^{\prime},
\end{aligned}
$$

where $\chi(\mathbf{r})=\varepsilon_{r}(\mathbf{r})-\varepsilon_{b r}$ denotes the permittivity contrast which vanishes outside $D \supset \Omega$, $G\left(\mathbf{r}, \mathbf{r}^{\prime}\right)$ is the two-dimensional free-space Green function and $k_{0}$ represents the vacuum wavenumber. For the sake of simplicity, the equations (Eq. 2) and (Eq. 3) are rewritten as

$$
E_{l}^{\mathrm{s}}=\mathbf{K} \chi E_{l}, \quad \text { and } \quad E_{l}=E_{l}^{\mathrm{i}}+\mathbf{G} \chi E_{l} .
$$

\section{Inverse scattering problem}

The inverse scattering problem is stated as finding the permittivity distribution in the box $D$ such that the corresponding scattered intensity predicted by the model via the coupling and the observation equation matches the data. Is proposed herein an iterative approach to solve this ill-posed and non-linear problem. The first step consists in the definition of a discrepancy criteria between the measured fields and the simulated ones. This criteria depends on the amount of available data, e.g., modulus and phase or modulus only. The derivative of this cost functional must then be explicitly obtained and it will be shown that it introduces an adjoint state equation where receivers act as sources with an amplitude mainly depending on the expression of the cost functional.

\section{A. Cost functional definition}

The parameter of interest, namely the contrast $\chi$, is gradually adjusted by minimizing a cost functional $\mathcal{J}(\chi)=\sum_{l=1}^{L} \mathcal{F}\left(E_{l}^{\mathrm{s}}(\chi)\right)$ suitably defined under the constraints of (Eq. 4). If 
both amplitudes and phase must be matched, the cost functional reads as

$$
\mathcal{J}(\chi)=\frac{1}{2} \sum_{l=1}^{L} w_{l}\left\|E_{l}^{\mathrm{obs}}-E_{l}^{\mathrm{s}}(\chi)\right\|_{\Gamma}^{2}
$$

where $E^{\text {obs }}$ correspond to the available measurements and $w_{l}$ to appropriate weight coefficients, for example, $w_{l}^{-1}=\left\|E_{l}^{\text {obs }}\right\|_{D}^{2}$. If scattered intensity must be matched, the cost functional reads as

$$
\mathcal{J}(\chi)=\frac{1}{2} \sum_{l=1}^{L} w_{l}\left\|I_{l}^{\mathrm{obs}}-\left|E_{l}^{\mathrm{s}}(\chi)\right|^{2}\right\|_{\Gamma}^{2}
$$

where $I^{\text {obs }}$ correspond to the available intensity measurements and $w_{l}^{-1}=\left\|I_{l}^{\text {obs }}\right\|_{\Gamma}^{2}$.

\section{B. Gradient expression}

This minimization problem under constraints can be reformulated using a Lagrangian functional $\mathcal{L} \mathrm{as}^{7}$

$$
\begin{aligned}
\mathcal{L}\left(\chi, E^{\mathrm{s}}, E, U^{\mathrm{s}}, U\right)= & \sum_{l=1}^{L}\left\{\mathcal{F}\left(E_{l}^{\mathrm{s}}\right)\right. \\
& +<U_{l}^{\mathrm{s}} \mid E_{l}^{\mathrm{s}}-\mathbf{K} \chi E_{l}>_{\Gamma} \\
& \left.+<U_{l} \mid E_{l}-E_{l}^{\mathrm{i}}-\mathbf{G} \chi E_{l}>_{D}\right\}
\end{aligned}
$$

where $\chi$ is the unknown contrast, $\mathcal{F}$ is the cost function to minimize, $E^{\mathrm{s}}$ and $E$ correspond to the simulated scattered and total fields, $U^{s}$ and $U$ are Lagrange multipliers, $<\mid>_{\Gamma}$ is the scalar product on $\Gamma\left(<u \mid v>_{\Gamma}=\int_{\Gamma} u^{*}(\mathbf{r}) v(\mathbf{r}) d \mathbf{r}\right)$ and $<\mid>_{D}$ the scalar product on $D$ $\left(<u \mid v>_{D}=\int_{D} u^{*}(\mathbf{r}) v(\mathbf{r}) d \mathbf{r}\right)$. This Lagrangian is used to express first-order and secondorder conditions for a local minimizer, which are linked to the existence of a saddle-point. This saddle-point provides an efficient way to compute the gradient of the cost functional by 
introducing an adjoint field. The adjoint field, due to the reciprocity principle, is equivalent to the direct field where receivers act are sources with an amplitude linked to the cost functional expression

$$
P_{l}=P_{l}^{\mathrm{i}}+\mathbf{G} \chi P_{l} \quad \text { and } \quad P_{l}^{\mathrm{i}}=-\mathbf{K}^{t} \overline{\nabla \mathcal{F}\left(E_{l}^{\mathrm{s}}\right)}
$$

If both amplitudes and phase must be matched, the incident adjoint field is given by

$$
P_{l}^{\mathrm{i}}=w_{l} \mathbf{K}^{t}\left(\overline{E_{l}^{\mathrm{obs}}-E_{l}^{\mathrm{s}}}\right)
$$

If scattered intensity must be matched, the sources for the adjoint problem read as

$$
P_{l}^{\mathrm{i}}=2 w_{l} \mathbf{K}^{t} \overline{E_{l}^{\mathrm{s}}}\left(I_{l}^{\mathrm{obs}}-\left|E_{l}^{\mathrm{s}}\right|^{2}\right)
$$

Therefore the adjoint method is a very convenient way for computing derivatives for several types of cost functional.

It can be shown (Sec. A) that the gradient of the cost functional is given by

$$
<\nabla \mathcal{J}(\chi)\left|\delta \chi>_{D}=-\Re e<\sum_{l=1}^{L} \overline{E_{l} P_{l}}\right| \delta \chi>_{D}
$$

In the case of intensity measurements, this gradient shows the ambiguity of the cost functional. On one hand, the cost functional can be reduced if the computed field is close to the measured field. On the other hand, the cost functional can be reduced if the size of the scatterer is very small and we can neglect its contribution. In that case, the adjoint field is null as well as the gradient.

\section{Gradient approximation}

The gradient evaluation requires the computation of two forward problems. The first one computes the direct field $E_{l}$ as the second one, where the receivers act as sources with a 
prescribed amplitude, provides the adjoint field $P_{l}$. It might be interesting, in order to save some computational time, to perform some approximations such as the Born approximation.

Three cases can be considered: (i) no approximation is done for the direct and adjoint field computation (noted as the FulL-Full case in the following), (ii) Born approximation is only made for the adjoint field computation (FULL-BORN) and (iii) finally the Born approximation is applied for both fields (BORN-BORN). In the last case, the gradient is identical to the one which would be obtained by assuming from the beginning that the Born approximation was valid. As expected, the way the gradient is computed will have an effect on the minimization process as it will be highlighted in Sec. 5 with some numerical examples.

\section{Minimization scheme}

Once the discrepancy criteria has been defined and its derivative computed, a minimization algorithm can then be applied, which can be specified according to the a priori information available. For example, if the permittivity profile of the unknown obstacle is assumed to be continuous, a standard conjugate-gradient type algorithm can be used. If, on the contrary, one is interested in looking at homogeneous by part obstacles, this a priori information can be introduced via a level-set formulation where the cost functional derivative is still needed. In all cases, the initial guess selection is a key point for the convergence of the minimization process.

\section{A. Initial guess selection}

The initial guess computation is based on topological asymptotic expansion results ${ }^{9}$. The topological derivative aims at introducing some small dielectric balls of constant permittivity 
$\varepsilon_{r}$ into a known background of permittivity $\xi(\mathbf{r})$. These balls induce variations on the electromagnetic fields which are expressed via a topological asymptotic expansion formula. Let us denote by $B_{\rho}$ a small dielectric ball of size $\rho|B|$ centered at point $\mathbf{r}(|B|$ is the measure of a reference ball $B$ ). This means that $\mathbf{r} \in B_{\rho} \subset B_{\rho^{\prime}}$ if $0<\rho<\rho^{\prime}<1$. The topological asymptotic expansion of our cost function can then be expressed by ${ }^{10}$

$$
\begin{aligned}
& \mathcal{J}\left\{\chi=\left(\varepsilon_{r}-\varepsilon_{b r}\right) \mathbb{1}_{B_{\rho}}\left(\xi-\varepsilon_{b r}\right) \mathbb{1}_{D \backslash B_{\rho}}\right\} \\
- & \mathcal{J}\left\{\chi=\left(\xi-\varepsilon_{b r}\right) \mathbb{1}_{D}\right\} \\
= & -\rho^{2} \Re e\left(\varepsilon_{r}-\varepsilon_{b r}\right) k_{0}^{2}|B|\left(\sum_{l=1}^{L} E_{l} P_{l}\right)+o\left(\rho^{3}\right)
\end{aligned}
$$

where $\mathbb{1}$ is the conventional characteristic function, $E_{l}$ (resp. $P_{l}$ ) verifies (Eq. 3) (resp. Eq. 9) with $\chi(\mathbf{r})=\xi(\mathbf{r})-\varepsilon_{b r}, \forall \mathbf{r} \in D$. This topological derivative provides therefore information where to place balls such that the cost functional is reduced and is directly linked to the topology of the scatterers. In fact, if we assume that $\xi=\varepsilon_{b r}$, this gradient is no more than the first step of the inversion process, as expressed in (Eq. 11), assuming that there is no initial guess.

Using this topological derivative, as we do not know the value of $\varepsilon_{r}$, we construct the initial guess with

$$
\chi_{0}(\mathbf{r})=\eta \Re e \sum_{l=1}^{L} E_{l}(\mathbf{r}) P_{l}(\mathbf{r})
$$

where $\eta$ is a constant defined such that $\mathcal{J}\left(\chi_{0}\right)$ is minimal. The fields $E_{l}$ and $P_{l}$ are the direct and adjoint fields computed for $\chi(\mathbf{r})=\xi-\varepsilon_{b r}, \forall \mathbf{r} \in D$, with $\xi$ very close from $\varepsilon_{b r}$. It would have been more natural to use $\chi=0$ on the entire test domain $D$ (which would have correspond to $\Omega=\emptyset$ ) but then, due to definition of the cost functional for intensity 
measurements, the adjoint field would have been null as well as the topological derivative.

If a priori information on the nature of the scatterer is given, such as the obstacle is homogeneous, a truncation at mid value is performed to obtain a binary image.

\section{B. Retrieval of an inhomogeneous profile}

If no a priori information is available on the nature of the scatterer, a sequence $\left\{\chi_{n}\right\}$ is built up iteratively according to the following relation

$$
\chi_{n}=\chi_{n-1}+\alpha_{n} d_{n}
$$

where $d_{n}$ is an updating direction and $\alpha_{n}$ a weight that is determined at each iteration step by minimizing the cost functional $\mathcal{J}\left(\chi_{n}\right)$ (Eq. (6)). During the local search for $\alpha_{n}$, the field $E$ remains fixed to the value obtained at previous iteration. As a search direction $d_{n}$, the authors take a Polak Ribière conjugate direction

$$
d_{n}=g_{n}+\gamma_{n} d_{n-1}, \quad \gamma_{n}=\frac{<g_{n} \mid g_{n}-g_{n-1}>_{D}}{\left\|g_{n-1}\right\|_{D}^{2}}
$$

where $g_{n}$ is the gradient of $\mathcal{J}(\chi)$ with respect to $\chi$. As described in Sec. $3 \mathrm{C}$, this gradient can be exactly computed or approximated.

\section{Retrieval of a binary profile}

As the nonlinear inverse problem stated above is highly ill-posed, all available information is useful to improve the quality of the reconstructions. In some cases, it is possible to assume that the dielectric properties of the obstacle are known and furthermore that this obstacle is homogeneous. The contrast of permittivity will then be a binary function of the following 
form:

$$
\chi(\mathbf{r})=\varepsilon_{r}-\varepsilon_{b r} \quad \mathbf{r} \in \Omega, \quad \chi(\mathbf{r})=0 \quad \mathbf{r} \notin \Omega
$$

where $\varepsilon_{r}$ is known and constant. In this approach, which is reduced to a shape optimization problem, the parameter of interest, namely the shape $\Omega$, is gradually adjusted by minimizing the same cost functional as previously under the constraints of (Eq. 2) and (Eq. 3). An sequence of shapes $\left\{\Omega_{n}\right\}$ is constructed in order to minimize the cost functional $\mathcal{F}\left(\Omega_{n}\right)$. For doing so, several elements are necessary : (i) the shape representation, (ii) the computation of the derivative of the cost functional according to shape, (iii) and the construction of the iterative sequence. To represent the shape, let us introduce an auxiliary function called a level-set function $\phi$ such that

$$
\Omega=\{\mathbf{r} \in D \text { s.t. } \phi(\mathbf{r})<0\} .
$$

This representation handles naturally all topological changes such as fusion or separation and does not require to know in advance the number of scatterers as well as their centers positions. The derivation of the cost functional $\mathcal{J}$ which now depends on $\phi$ must then be done according to this level-set representation, to obtain

$$
\begin{aligned}
<\nabla \mathcal{J}(\phi) \mid \delta \phi>_{D}= & -\Re e\left(\varepsilon_{r}-\varepsilon_{b r}\right) \\
& <\delta(\phi)|\nabla \phi| \sum_{l=1}^{L} \overline{E_{l} P_{l}} \mid \delta \phi>_{D}
\end{aligned}
$$

where $\delta(\phi)$ corresponds to the one-dimensional Dirac delta function concentrated on the interface $\phi=0$, i.e., the interface $\partial \Omega$. As described in Sec. $3 \mathrm{C}$, this gradient can be exactly computed or approximated. An artificial time variable $t$ is introduced and the minimization 
is done by finding the steady state solution of

$$
\phi_{t}=-\nabla \mathcal{J}(\phi)
$$

assuming that the $\delta(\phi)$ function is extended everywhere in $D$ with value 1 . This equation is solved using the Osher-Sethian numerical scheme described in Ref. 11.

\section{Numerical experiments}

In this section we report examples of reconstructions of dielectric samples to illustrate the efficiency of the inversion algorithms presented in the previous sections. In all cases, synthetic data are generated thanks to a fast forward solver described in details in Ref. 12. This forward solver is based on a second-order accurate space-discretisation which is capable of handling homogeneous as well as inhomogeneous profiles. The convolution-type structure of the integral equation is exploited and solved via a Conjugate Gradient-Fast Fourier Transform (CG-FFT) method. Moreover, a special extrapolation procedure is used, by "marching-on-in" source position, to generate accurate initial estimates for the CG method to reduce the computation time. On the contrary, the inversion solver is based on a standard method of moment without any use of the CG-FFT method ${ }^{12}$. This solver is needed for computing both the internal and adjoint fields. The dielectric permittivity as well as the electromagnetic field are interpolated by piecewise constant basis functions with collocation point test functions.

The receivers as well as the sources are assumed to be infinite lines located on a circle $\Gamma$ of radius $1.5 \lambda, \lambda$ being the wavelength in the vacuum. In addition, we consider 64 sources and receivers evenly distributed on the measurement circle $\Gamma$. The mesh size of the forward 
solver to generate data is $\lambda / 64$. The investigated domain $D$ is a square box of side size $2 \lambda$, subdivided for numerical purposes into 30 square cells, leading thus to a mesh size of $\lambda / 15$ for inversion schemes. Consequently, the mesh size used in the inversion is different from the one used to generate data, preventing any "inverse crime". In all the following examples, the initial guess is chosen as described in Sec. $4 \mathrm{~A}$ with an initial contrast of $\chi=\xi-\varepsilon_{b r}=1.01$. For such contrast value, the Born approximation is applicable. Finally, all iterative schemes have been conducted up to the 512-th iteration, to ensure that convergence, if there is one, is achieved. In all cases, the evolution of the cost function is presented. By letting the inversion algorithm runs, we then have a good indication of the convergence speed, the discrepancy accuracy and the trends of the methods. In particular, we can check if we reached a plateau or if the algorithm is unstable.

\section{A. Reconstruction of spatially homogeneous profiles}

1. The HомоCYL16 object

As a first example, we consider two circular homogeneous cylinders of radii $a_{1}=0.15 \lambda$ and $a_{2}=0.3 \lambda$, and of relative permittivity $\varepsilon_{r}=1.6$. The small cylinder is located at $(-0.2 \lambda, 0.2 \lambda)$ while the other cylinder is located at $(-0.3 \lambda,-0.3 \lambda)$. Henceforth, this object under test is referred as HomoCYL16 object.

To emphasize the influence of the phase information, two initial estimates obtained with the same topological expansion method are plotted in Fig. 2 for the HomoCyL16 object. In Fig. 2(a), only modulus information is used as in Fig. 2(b), modulus and phase are taken into account. It is clear that the phase contains important topological information. Therefore, 
by using modulus only data, we are more penalized in the reconstruction process as using a full scattered field.

Fig. 3 present the reconstructed contrast $\chi$ within the investigated domain $D$, using the inversion algorithm described in Sec. 3 B, for various choices of descent direction. Clearly, the best result, Fig. 3(a), is obtained when both the internal and the adjoint fields are computed without assuming the Born approximation (Full-Full case). Comparing the reconstructed profiles to the actual one, Fig. 4, shows that not only the shape is well retrieved but also the refractive index. Surprisingly, the others cases, in particular the BORN-BORN case (Fig. 3(e)), lead to relatively accurate reconstruction of the target under test. We emphasize that the object under test is of characteristic dimension about $\lambda$ and of dielectric contrast of $\chi=0.6$, for which the Born approximation is not valid.

The evolution of the cost functional in the case of FULL-Born (Fig. 3(d)) exhibits a minimum around the iteration 128. Indeed, the corresponding image, not plotted here, is almost as good as Fig. 3(a). After this iteration, the cost functional starts to increase again to reach a plateau whose corresponding image is presented in Fig. 3(c). This is due to the fact that, near the minimum, exact gradient computation is of high importance, especially as it is of very small value and as numerical noise might cause the divergence of the iterative process. This divergence shows the importance of a correct computation of the gradient. In all cases, such behavior is not observed for the two other schemes, where the computation of the forward and adjoint fields are consistent.

The same behavior can be observed using a priori information on the nature of the scatterers by means of the level-set scheme described in Sec. 4 C. Fig. 5 show the reconstructed 
images obtained after 512 iterations when using different ways of computing the gradient. The initial guess was computed as previously and truncated at mid value to obtain a binary image. Again, Full-Full and BORn-BORN cases provide very satisfactory results compared to the Full-BORN case. The oscillations in the cost functional appear when the size of the image changes are of the order of the cell size.

\section{The HomoCyL20 object}

Consider the same two cylinders slightly closer and of relative permittivity $\varepsilon_{r}=2.0$ instead of $\varepsilon_{r}=1.6$. The small cylinder is now located at $(-0.15 \lambda, 0.15 \lambda)$. From now on, this object is referred as HomOCYL20. Fig. 6 present results of the reconstructed contrast profile using the conjugate gradient algorithm for various choices of the gradient. Contrary to the preceding case, the convergence in the case of FulL-FulL (Fig. 6(a)) is slow. The best result is obtained for the case of FULL-FulL, while for the other cases, FulL-BorN and BORN-BORN, the reconstructed targets are blurred and melted with artifacts. Fig. 7 shows quantitative comparisons between reconstructed targets and the actual ones along diameters of the cylinders.

As the level-set algorithm use very strong a priori on the nature of the scatterer, the reconstructions are improved for this obstacle and the artifacts disappear as shown in Fig. 8. This effect is also partly due to multiple scattering effects ${ }^{13}$ which is fully taken into account when using a FULL-FULL approach for the gradient computation and explains how the small scatterer is well reconstructed. Again, the FulL-BORn case provides the worst result and starts to diverge after a while. On the other hand, this case was the first to converge towards 
an acceptable solution.

From these two examples, one may conclude that the inversion in the FuLL-FulL case is more accurate than the two other cases. It requires more computation time than the BORN-BORN case but it takes into account the multiple scattering effect. Compared to the Full-BORN case, the extra computational burden is minimal, as nearly everything has already been computed to obtain the internal field, and the results are more satisfactory.

\section{B. Reconstruction of spatially continuous profiles}

We consider now two inhomogeneous profiles, denoted as LUNEBERG and INHOMOSin. These two profiles consist of an inhomogeneous circular cylinder of radius $a=0.7 \lambda$, located at $(0.15 \lambda,-0.15 \lambda)$. The contrasts within the objects are radially varying. For the profile LUNEBERG, the contrast is of the form $\chi(\rho)=1-(\rho / a)^{2}$, while for the object InHomoSin, the contrast is of the form $\chi(\rho)=\sin ^{2}(\pi \rho / a)$, where $\rho$ denotes the radial coordinate in the frame of the center of the cylinder. These profiles are spatially continuous and cannot be represented by a binary level-set representation neither by the extended one for the representation of multiple constitutive materials as suggested in Ref. 14. In addition, these obstacles present internal fields which are strongly different from the incident field as shown in Fig. 9. The object LuneBerg is known as an ideal two-dimensional Luneberg lens. For the object InHomoSin, it is predicted the presence of whispering-gallery modes that propagates along the interior boundary of the cylinder. ${ }^{15}$ 
1. Inversion from noiseless data

Figs. (10) and (11) present results of the reconstruction of the target LUNEBERG and INHomoSin, respectively. In all cases, the support of the object under test is well retrieved. However, in the case of the computation under the assumption of the Born approximation for both the internal field and the adjoint field, the reconstructed contrast profile is meaningless as clearly shown in Fig 12. A perfect reconstruction is obtained for the FULL-FULL case for both profiles.

2. Inversion from noisy data

In this section are presented results of inversion from noisy data. We restrict ourselves to the case of inhomogeneous targets (LunEBERG and InHomoSin) targets, in view of the fact that no prior information is introduced. The case of homogeneous targets is expected to be more robust against the presence of noise in data. Uniform white noise has been added to the simulated intensity data. Hence, the input data used for the inversion are corrupted according to the following relation

$$
\tilde{I}_{l}^{\mathrm{obs}}(\mathbf{r})=(1+b u) I_{l}^{\mathrm{obs}}(\mathbf{r})
$$

where $\tilde{I}_{l}^{\text {obs }}$ denotes the corrupted data, $u \in[-1,1]$ is a random number, $b$ monitors the level of noise. Fig. 13 presents the result of the inversion for the LunEBERG and the InHomoSin targets, respectively. For these numerical experiments, the level of noise $b$ is as high as $10 \%$. It is clearly shown (see Fig. 14) that the FULL-FuLL scheme is more robust against the presence of noise in the data than the two others inversion schemes (FULL-BORN and BORN-BORN). 


\section{Conclusion}

We have examined two configurations of practical interest of inverse scattering from intensity measurements. The first one was related to the retrieval of heterogeneous objects, while the second was more specific to homogeneous ones. A cost functional criterion has been defined and minimized to compute the best available estimate. We have shown that the gradient computation is similar to the one that would have been obtained when using modulus and phase information. Indeed, this gradient is obtained by combining an internal field and an adjoint field where the receivers act as sources with a prescribed amplitude which differs according to the available data. We have also shown that this gradient can be used as an initial guess, based on topological derivative results.

We have explored the Born approximation for the internal and the adjoint field and numerical examples have shown that the inversion in the FULL-FulL case was more accurate than using a Born approximation for the adjoint and/or the internal field. This behavior has been observed for both homogeneous and heterogeneous obstacles. Even if the computational burden is slightly higher in the FULL-FulL case, this can be significantly reduced by using fast forward solvers. In addition, the FULL-FULL scheme is more robust against the presence of noise than the two other ones.

The numerical examples have also shown the influence of a priori information, in particular when the obstacle are homogeneous. In that case, the level-set representation provide final results where the boundaries of the obstacle are better resolved. It would be interesting to see what would be the extension of the inverse scattering problem with intensity measurements with homogeneous by parts obstacles using the ideas of Ref. 14 . 
It has been also shown that the gradient of the cost functional is null if the initial guess is a flat background, a situation which does not appear when using modulus and phase data. In order to compute properly the topological asymptotic expansion and use it as an initial guess, it would be interesting to look at second-order derivative following the ideas of Ref. 16.

Finally, this work can easily be extended to the case of obstacles placed on a substrate, which is the typical configuration of optical diffraction setups. The main difference will rely in the Green functions which will have to take into account the interfaces. The next step will be to handle real data sets.

\section{Appendix A: Gradient computation}

The parameter of interest, here the contrast $\chi$, must minimize a properly defined cost functional $\mathcal{J}(\chi)$ (see (Eq.5) and (Eq. 6)), under the constraints of (Eq. 4). Let us assume furthermore that the cost functional is such that, for all $\delta E^{\mathrm{s}}$,

$$
\begin{aligned}
\mathcal{F}\left(E^{\mathrm{s}}+\delta E^{\mathrm{s}}\right)= & \mathcal{F}\left(E^{\mathrm{s}}\right)+\Re e<\nabla \mathcal{F}\left(E^{\mathrm{s}}\right) \mid \delta E^{\mathrm{s}}>_{\Gamma} \\
& +o\left(\left\|\delta E^{\mathrm{s}}\right\|_{\Gamma}\right)
\end{aligned}
$$

If for example, amplitude and phase measurements must be matched, this means $\nabla \mathcal{F}\left(E_{l}^{\mathrm{s}}\right)=-w_{l}\left(E_{l}^{\mathrm{obs}}-E_{l}^{\mathrm{s}}\right)$. If intensity measurements must be matched, this means $\nabla \mathcal{F}\left(E_{l}^{\mathrm{s}}\right)=-2 w_{l} E^{\mathrm{s}}\left(I_{l}^{\mathrm{obs}}-\left|E_{l}^{\mathrm{s}}\right|^{2}\right)$.

Let us denote by $\mathcal{L}$ the Lagrangian functional defined in (Eq. 7). It can be noticed that, if the fields $E^{\mathrm{s}}$ and $E$ both satisfy the forward equations, then

$$
\mathcal{L}\left(\chi, E^{\mathrm{s}}(\chi), E(\chi), U^{\mathrm{s}}, U\right)=\mathcal{J}(\chi) \quad \forall U^{\mathrm{s}}, \forall U
$$


If we derive this equation in the $\delta \chi$ direction, we get

$$
\begin{aligned}
< & \nabla \mathcal{J}(\chi)\left|\delta \chi>_{D}=<\partial_{\chi} \mathcal{L}\left(\chi, E^{\mathrm{s}}, E, U^{\mathrm{s}}, U\right)\right| \delta \chi>_{D} \\
& +<\partial_{E^{\mathrm{s}}} \mathcal{L}\left(\chi, E^{\mathrm{s}}, E, U^{\mathrm{s}}, U\right) \partial_{\chi} E^{\mathrm{s}} \mid \delta \chi>_{D} \\
& +<\partial_{E} \mathcal{L}\left(\chi, E^{\mathrm{s}}, E, U^{\mathrm{s}}, U\right) \partial_{\chi} E \mid \delta \chi>_{D}
\end{aligned}
$$

The quantities $U^{\mathrm{s}}$ and $U$ are chosen in order to eliminate the last terms in the summation, i.e., they must satisfy the following adjoint equations

$$
\begin{array}{ll}
<\partial_{E^{\mathrm{s}}} \mathcal{L}\left(\chi, E^{\mathrm{s}}, E, U^{\mathrm{s}}, U\right) \mid \delta E^{\mathrm{s}}>_{\Gamma}=0 & \forall \delta E^{\mathrm{s}} \\
<\partial_{E} \mathcal{L}\left(\chi, E^{\mathrm{s}}, E, U^{\mathrm{s}}, U\right) \mid \delta E>_{D}=0 & \forall \delta E
\end{array}
$$

This implies that the Lagrangian coefficient $U_{l}^{\mathrm{s}}$ must satisfy the following equation

$$
U_{l}^{\mathrm{s}}=-\nabla \mathcal{F}\left(E_{l}^{\mathrm{s}}\right)
$$

Replacing into the second equation, combined with the reciprocity principle $\overline{\mathbf{G}}^{\dagger}=\mathbf{G}$, and using the notation $U_{l}=\overline{\chi P_{l}}$, this induces the adjoint state equation

$$
P_{l}=\mathbf{G} \chi P_{l}-\mathbf{K}^{t} \overline{\nabla \mathcal{F}\left(E_{l}^{\mathrm{s}}\right)}
$$

This equation is similar to the forward problem equation where only the incident field has changed. For the adjoint problem, the incident field is due to the receivers which act as sources with an amplitude specified by $\overline{\nabla \mathcal{F}\left(E_{l}^{\mathrm{s}}\right)}$.

Let us go back to the derivation in the $\delta \chi$ direction, computed at the saddle-point position. This means that

$$
\begin{aligned}
<\nabla \mathcal{J}(\chi) \mid \delta \chi>_{D} & =<\partial_{\chi} \mathcal{L}\left(\chi, E^{\mathrm{s}}, E, U^{\mathrm{s}}, U\right) \mid \delta \chi>_{D} \\
& =-\Re e<\sum_{l=1}^{L} \overline{E_{l} P_{l}} \mid \delta \chi>_{D}
\end{aligned}
$$


where $P_{l}$ satisfy the adjoint state equation.

\section{References}

1. V. Lauer, "New approach to optical diffraction tomography yielding a vector equation of diffraction tomography and a novel tomographic microscope," J. Microsc. 205, 165-176 (2002).

2. N. Destouches, C. A. Guérin, M. Lequime, and H. Giovannini, "Determination of the phase of the diffracted field in the optical domain. Application to the reconstruction of surface profiles," Opt. Commun. 198, 233-239 (2001).

3. G. Gbur and E. Wolf, "The information content of the scattered intensity in diffraction tomography," Information sciences 162, 3-20 (2004).

4. L. Crocco, M. D’Urso, and T. Isernia, "Inverse scattering from phaseless measurements of the total field on a closed curve," J. Opt. Soc. Am. A 21, 622-631 (2004).

5. T. Takenaka, J. N. Wall, H. Harada, and M. Tanaka, "Reconstruction algorithm of the refractive index of a cylindrical object from the intensity measurements of the total field," Microwave Opt. Tech. Lett. 14, 182-188 (1997).

6. M. Lambert and D. Lesselier, "Binary-constrainted inversion of a buried cylindrical obstacle from complete and phaseless magnetic fields," Inverse Probl. 16, 563-576 (2000).

7. F. James and M. Sepulveda, "Parameter identification for a model of chromatographic column," Inverse Probl. 10, 1299-1314 (1994).

8. A. Litman, D. Lesselier, and F. Santosa, "Reconstruction of a two-dimensional binary 
obstacle by controlled evolution of a level-set," Inverse Probl. 14, 685-706 (1998).

9. M. Masmoudi, J. Pommier, and B. Samet, "The topological asymptotic expansion for the Maxwell equations and some applications," Inverse Probl. 21, 547-564 (2005).

10. B. Samet, "L'analyse asymtotique topologique pour les équations de Maxwell et applications," Ph.D. thesis, Université Paul Sabatier, Toulouse (2004).

11. S. Osher and J. A. Sethian, "Fronts propagating with curvature-dependent speed: algorithms based on Hamilton-Jacobi formulations," J. Comput. Physics 79, 12-49 (1988).

12. Z. Q. Peng and A. G. Tijhuis, "Transient scattering by a lossy dielectric cylinder: marching-on-in frequency approach," JEWA 7, 739-763 (1993).

13. K. Belkebir, P. C. Chaumet, and A. Sentenac, "Influence of multiple scattering on three-dimensional imaging with optical diffraction tomography," J. Opt. Soc. Am. A 23, 586-595 (2006).

14. A. Litman, "Reconstruction by level sets of n-ary scattering obstacles," Inverse Probl. 21, S131-S152 (2005).

15. A. G. Tijhuis, "Angularly propagating waves in a radially inhomogeneous, lossy dielectric cylinder and their connection with natural modes," IEEE Trans. Antennas Propagat. 34, 813-824 (1986).

16. J. Sokolowski and A. Zochowski, "On topological derivative in shape optimization," Tech. Rep. RR-3170, INRIA (1997). 


\section{List of figures}

Fig. 1: Geometry of the problem. A two-dimensional cylinder with cross-section $\Omega$ and of permittivity contrast $\chi(x, y)$ is radiated by an electromagnetic source located on a circle $\Gamma$. The scattered intensity is assumed to be available on $\Gamma$.

Fig. 2: Initial guess using the topological asymptotic expansion results (a) with modulus only data; (b) with modulus and phase data. The object under test HomoCyL16 is constituted by two circular cylinders of contrast $\chi=0.6$. Black circles in the images correspond to boundaries of actual cylinders.

Fig. 3: Reconstructed contrast distribution using a conjugate gradient method, for the HomoCyL16 object. The updating direction $d_{n}$ involves a gradient derived from a solution of an adjoint problem. (a) Both the internal field and the adjoint field are computed accurately (FulL-Full case); (c) Same as in (a) but the evaluation of the adjoint field assumes the Born approximation (Full-Born case); (e) The Born approximation is assumed for both the internal field and for the adjoint field (Born-Born case). Curves (b), (d) and (f) represent the evolution in log-scale of the minimized cost functional with respect to the iteration steps for the reconstructions plotted in (a), (c) and (e), respectively.

Fig. 4: Comparisons between the reconstructed contrast presented in Fig. 3 and the actual one along the $x$-axis. Left column comparisons are presented along the line $y=-0.3 \lambda$, which corresponds to a cut along a diameter of the large cylinder of Fig. 3. The right column present comparisons along the line $y=0.2 \lambda$, which corresponds to a cut along a diameter of the small cylinder of Fig. 3. Full line corresponds to the actual profile, while the dotted line corresponds to the reconstructed one. (a) and (b) correspond to Fig.3(a). (c) and (d) 
correspond to Fig.3(c). (e) and (f) correspond to Fig.3(e).

Fig. 5: Same as in Fig. 3, but the inversion is performed using the level-set scheme described in Sec. 4C, where it is assumed that the permittivity contrast of targets under test is known.

Fig. 6: Same as in Fig. 3, but with the object under test HomOCyL20, which is constituted by circular cylinders of permittivity contrast $\chi=1$.

Fig. 7: Comparisons between the reconstructed contrast presented in Fig. 6 and the actual one along the $x$-axis. Left column comparisons are presented along the line $y=-0.3 \lambda$, which corresponds to a cut along a diameter of the large cylinder of Fig. 6. The right column present comparisons along the line $y \approx 0.15 \lambda$, which corresponds to a cut along a diameter of the small cylinder of Fig. 6. Full line corresponds to the actual profile, while the dotted line corresponds to the reconstructed one. (a) and (b) correspond to Fig. 6(a); (c) and (d) correspond to Fig.6(c); (e) and (f) correspond to Fig.6(e).

Fig. 8: Same as in Fig. 6, but the inversion is performed using the level-set scheme described in Sec. 4 C.

Fig. 9: Modulus of electromagnetic fields in the test domain $D$ for a source located at $(1.5 \lambda, 0)(a)$ Incident field; (b) Internal field of the object LunEBERG; (c) Internal field of the object INHOMOSIN.

Fig. 10: Reconstruction of the inhomogeneous object LunEBERG from noiseless data, using the conjugate gradient method described in Sec. (3 B). (a) FulL-Full case; (c) FulLBORn case; (e) BORn-BORn case. The second column of the figure present the evolution in log-scale of the minimized cost functional versus iteration steps which correspond to images 
plotted in the first column.

Fig. 11: Same as in Fig. 10 but with the object InhomoSin.

Fig. 12: Comparisons between the reconstructed profiles and the actual one along a horizontal line $y \approx-0.15 \lambda$ for the LuneBERG (first column) and InHomoSin objects (second column). Full line stands for the actual profile while the dotted line corresponds to the reconstructed one. (a)(b) Full-Full; (c)(d) Full-Born and (e)(f) Born-Born.

Fig. 13: Same as in Fig. 10 and Fig. 11 but with $10 \%$ additive noise in the data. First column corresponds to the LUNEBERG object while the second column corresponds to the INHOMOSin object.

Fig. 14: Same as in Fig. 12 but with $10 \%$ additive noise. 


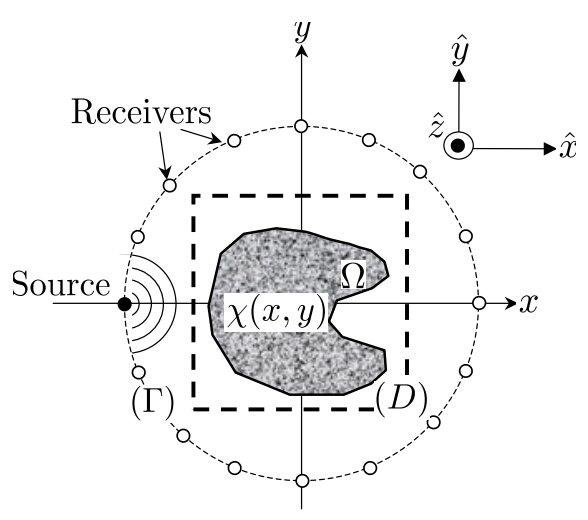

Fig. 1. Geometry of the problem. A two-dimensional cylinder with cross-section $\Omega$ and of permittivity contrast $\chi(x, y)$ is radiated by an electromagnetic source located on a circle $\Gamma$. The scattered intensity is assumed to be available on $\Gamma$. 

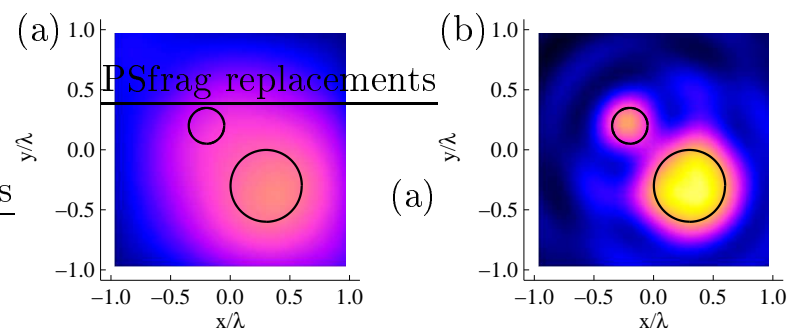

Fig. 2. Initial guess using the topological asymptotic expansion results (a) with modulus only data; (b) with modulus and phase data. The object under test HomoCyL16 is constituted by two circular cylinders of contrast $\chi=0.6$. Black circles in the images correspond to boundaries of actual cylinders. 

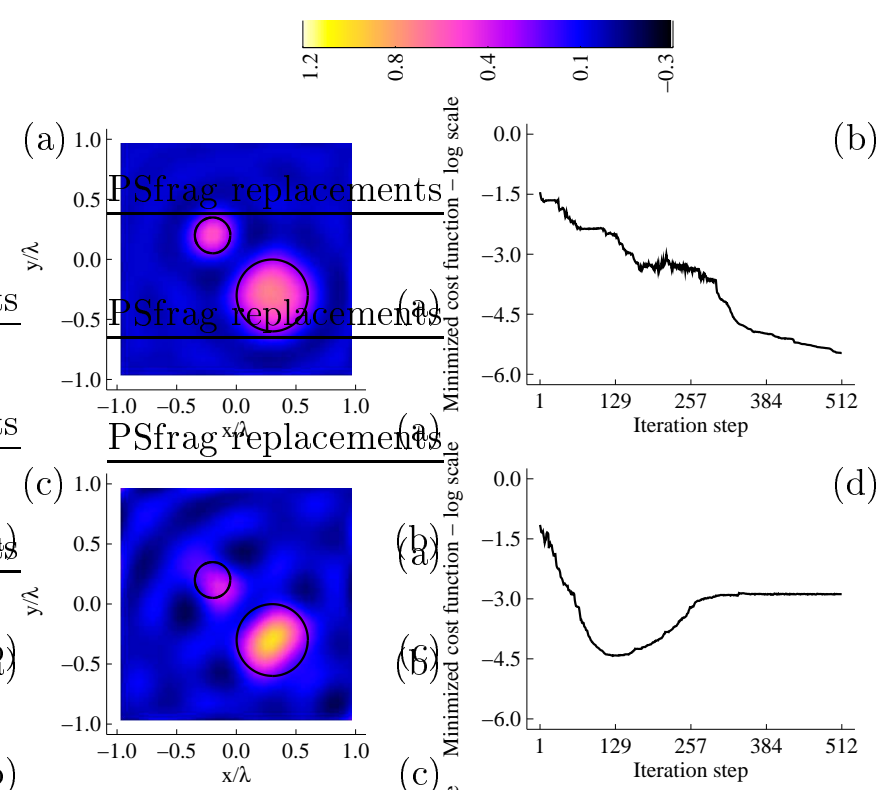

(b)
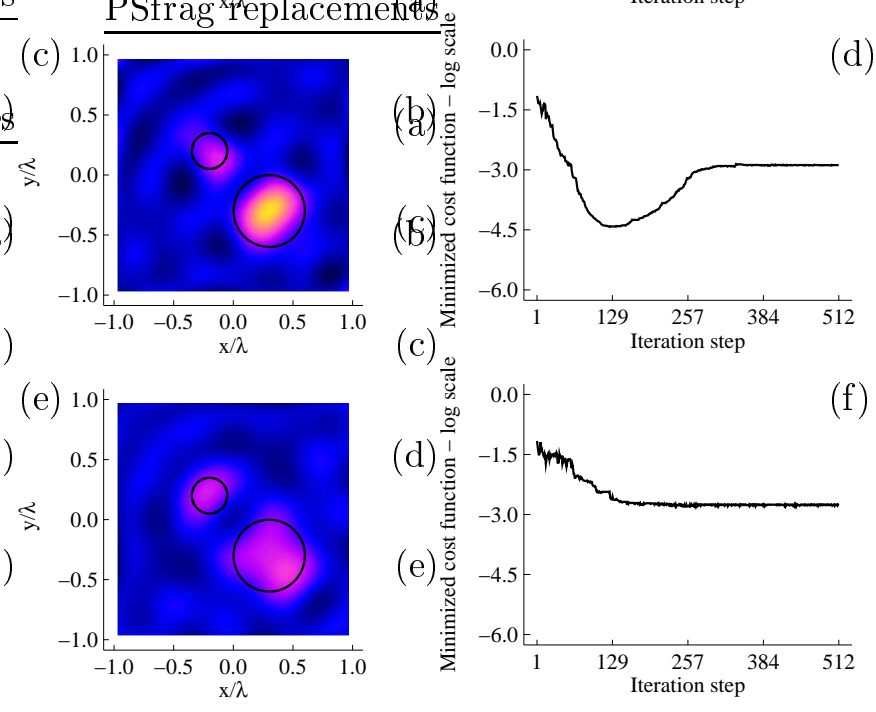

Fig. 3. Reconstructed contrast distribution using a conjugate gradient method, for the HoMOCYL16 object. The updating direction $d_{n}$ involves a gradient derived from a solution of an adjoint problem. (a) Both the internal field and the adjoint field are computed accurately (FulL-Full case); (c) Same as in (a) but the evaluation of the adjoint field assumes the Born approximation (Full-Born case); (e) The Born approximation is assumed for both the internal field and for the adjoint field (Born-Born case). Curves (b), (d) and (f) represent the evolution in log-scale of the minimized cost functional with respect to the iteration steps for the reconstructions plotted in (a), (c) and (e), respectively. 

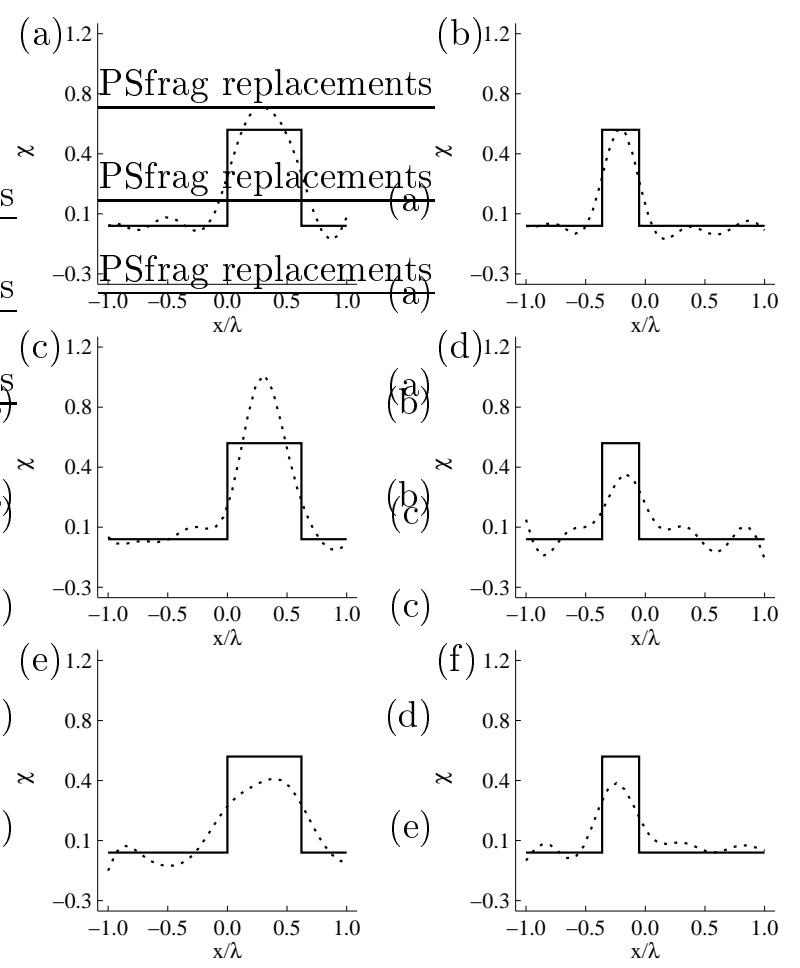

Fig. 4. Comparisons between the reconstructed contrast presented in Fig. 3 and the actual one along the $x$-axis. Left column comparisons are presented along the line $y=-0.3 \lambda$, which corresponds to a cut along a diameter of the large cylinder of Fig. 3 . The right column present comparisons along the line $y=0.2 \lambda$, which corresponds to a cut along a diameter of the small cylinder of Fig. 3. Full line corresponds to the actual profile, while the dotted line corresponds to the reconstructed one. (a) and (b) correspond to Fig.3(a). (c) and (d) correspond to Fig.3(c). (e) and (f) correspond to Fig.3(e). 

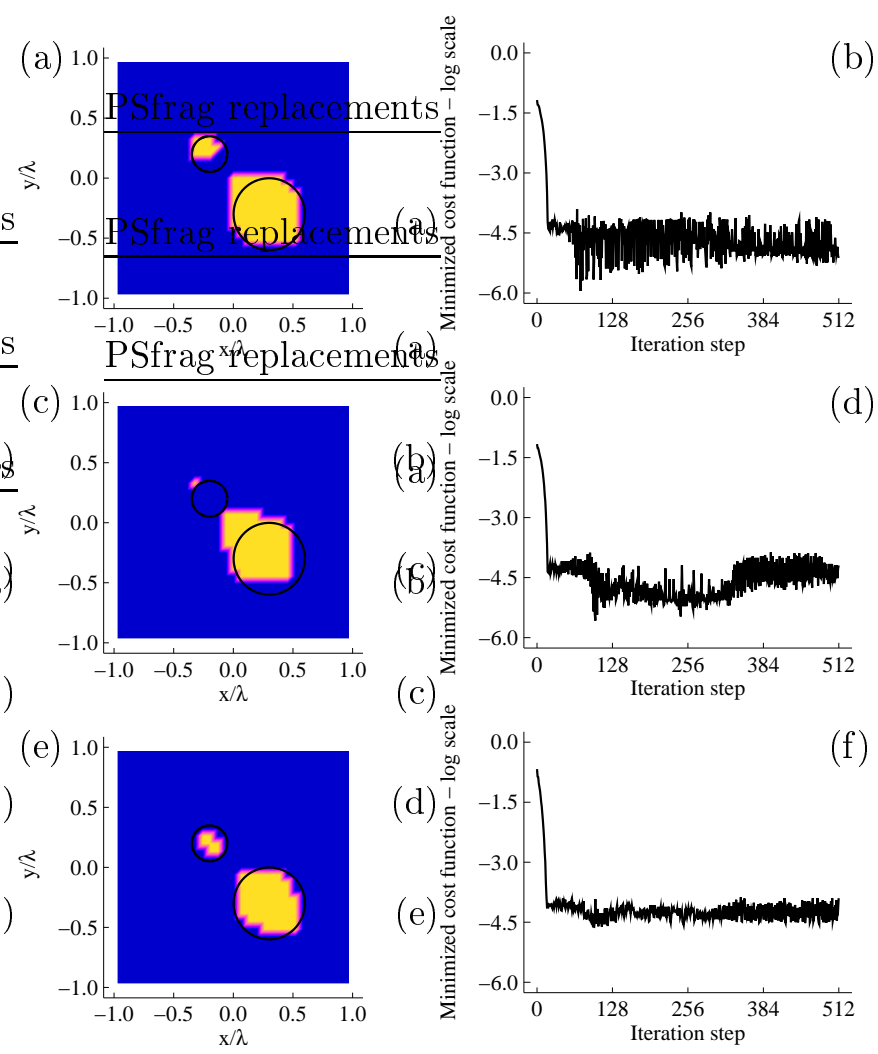

Fig. 5. Same as in Fig. 3, but the inversion is performed using the level-set scheme described in Sec. $4 \mathrm{C}$, where it is assumed that the permittivity contrast of targets under test is known. 

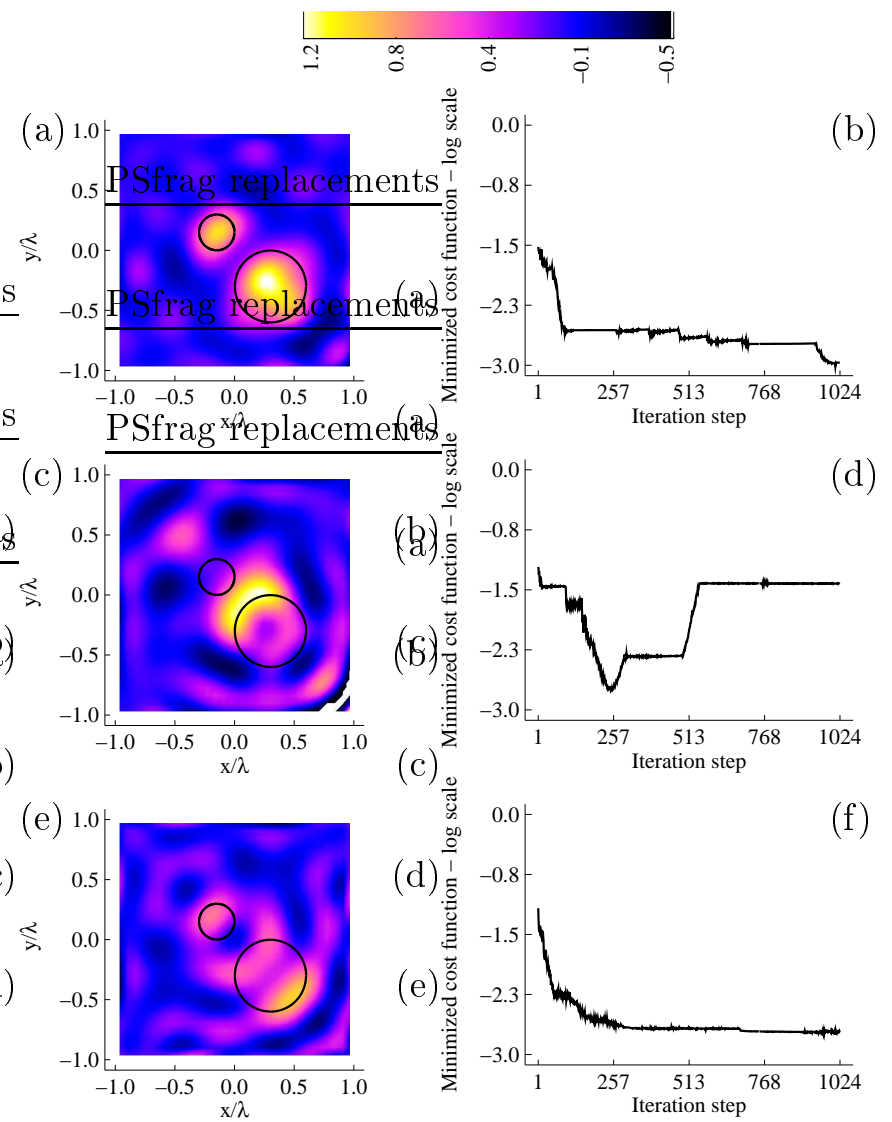

Fig. 6. Same as in Fig. 3, but with the object under test HomoCyL20, which is constituted by circular cylinders of permittivity contrast $\chi=1$. 

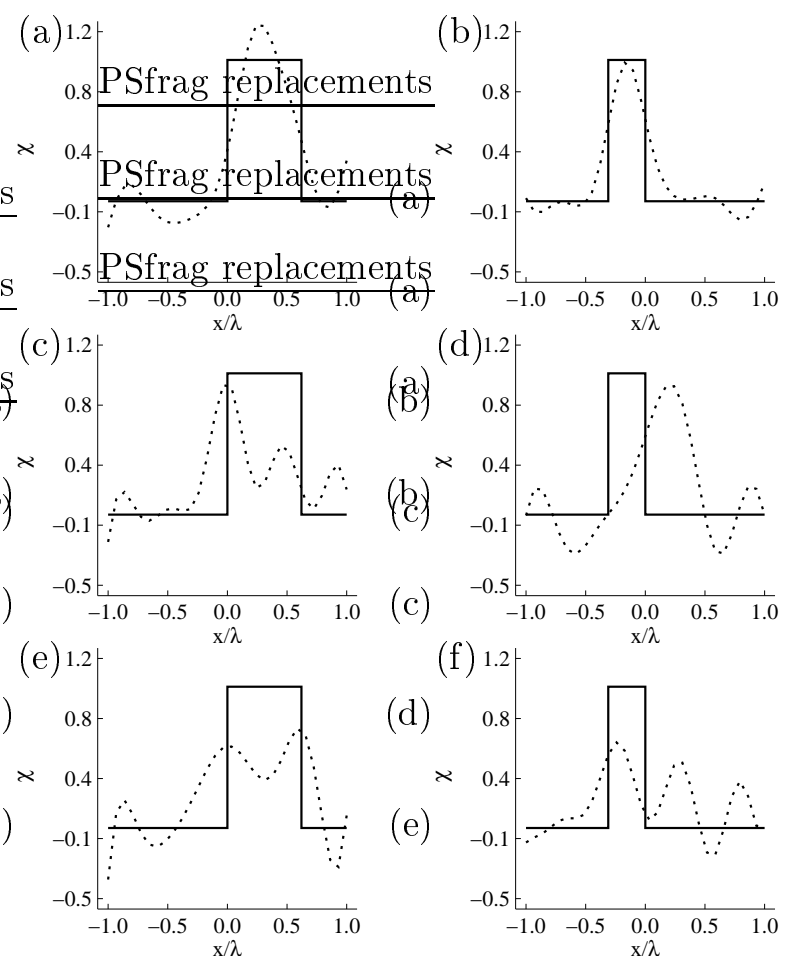

Fig. 7. Comparisons between the reconstructed contrast presented in Fig. 6 and the actual one along the $x$-axis. Left column comparisons are presented along the line $y=-0.3 \lambda$, which corresponds to a cut along a diameter of the large cylinder of Fig. 6. The right column present comparisons along the line $y \approx 0.15 \lambda$, which corresponds to a cut along a diameter of the small cylinder of Fig. 6. Full line corresponds to the actual profile, while the dotted line corresponds to the reconstructed one. (a) and (b) correspond to Fig. 6(a); (c) and (d) correspond to Fig.6(c); (e) and (f) correspond to Fig.6(e). 

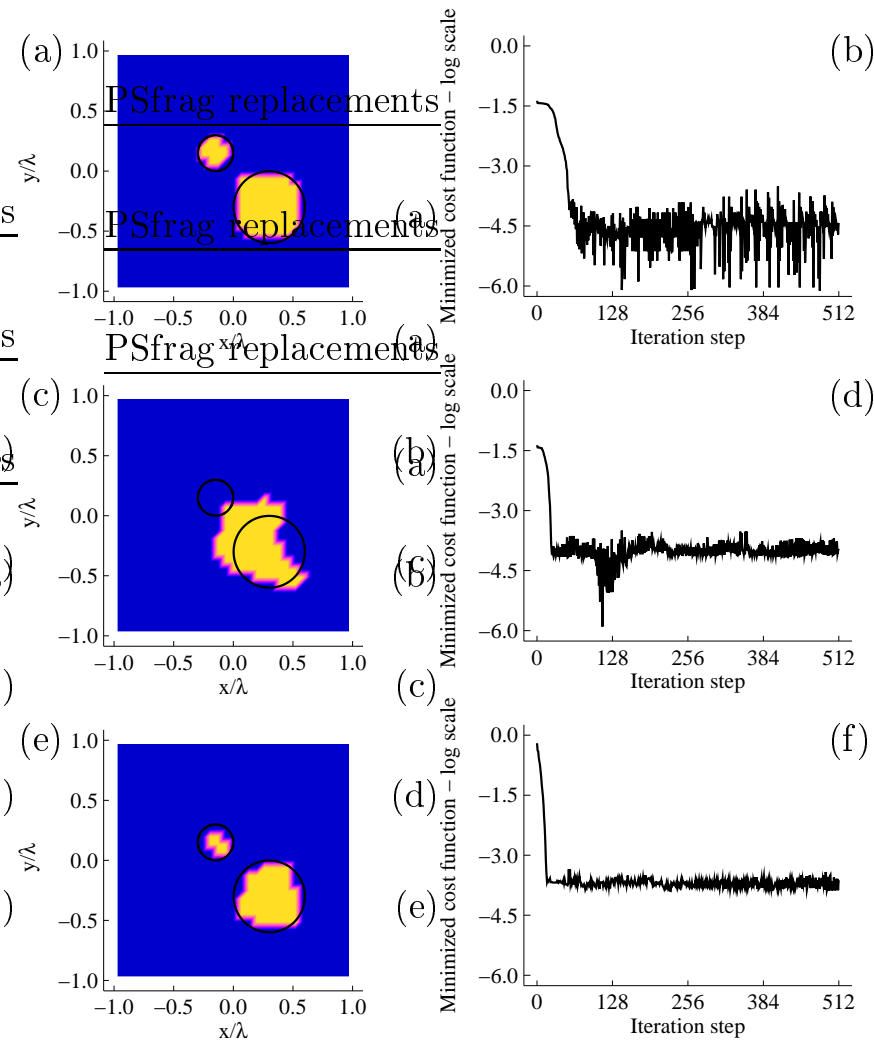

Fig. 8. Same as in Fig. 6, but the inversion is performed using the level-set scheme described in Sec. 4 C. 

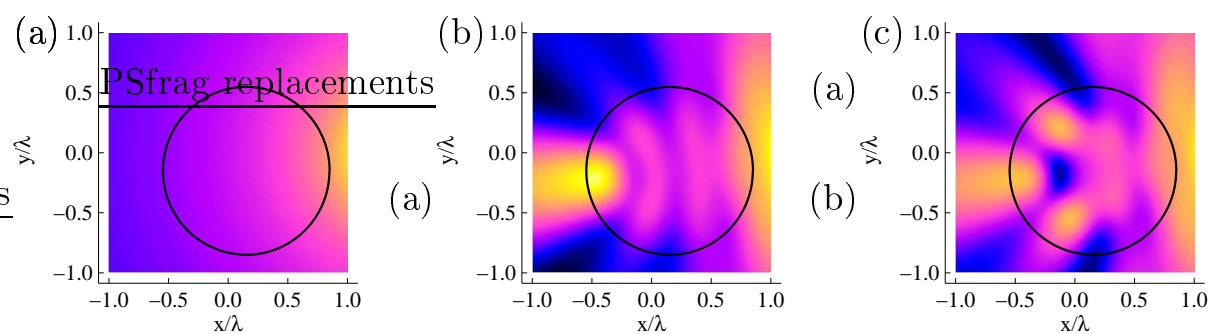

Fig. 9. Modulus of electromagnetic fields in the test domain $D$ for a source located at $(1.5 \lambda, 0)$ (a) Incident field; (b) Internal field of the object LunEBERG; (c) Internal field of the object INHOMOSin. 

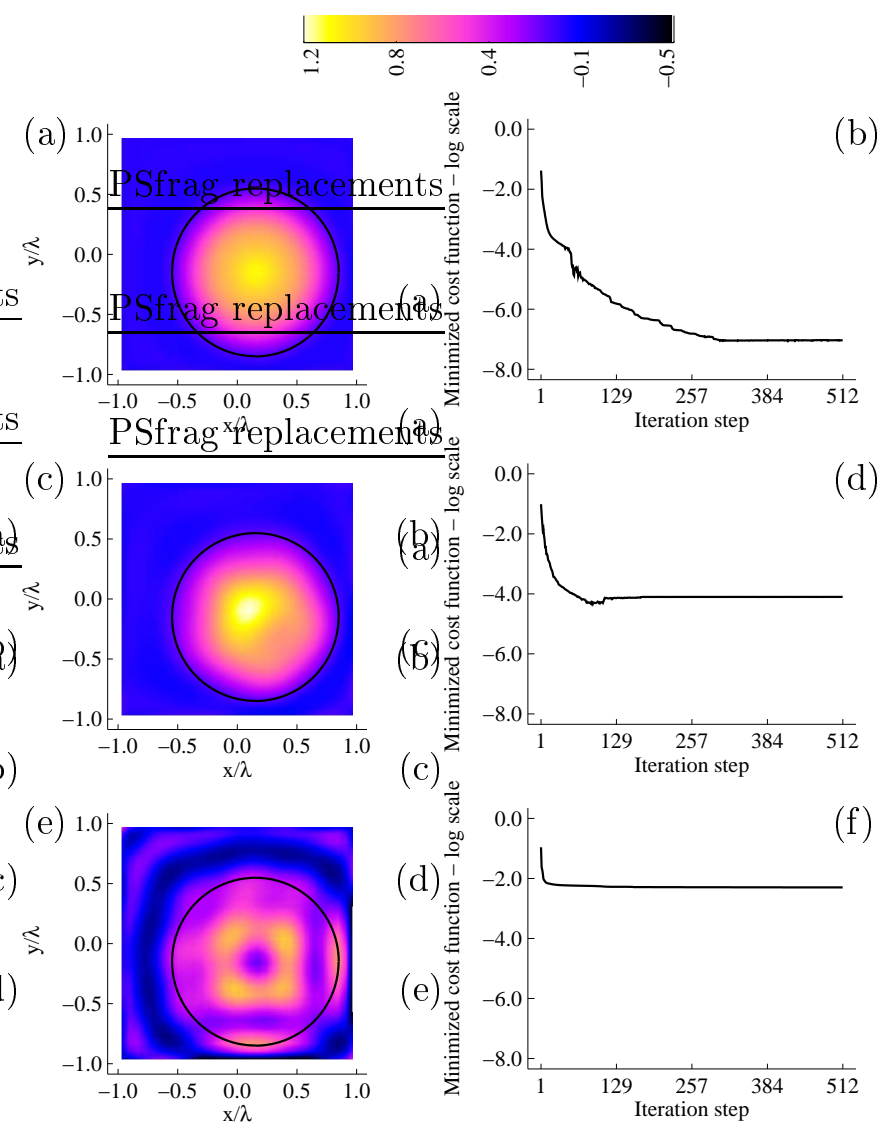

Fig. 10. Reconstruction of the inhomogeneous object LunEBERG from noiseless data, using the conjugate gradient method described in Sec. (3B). (a) Full-Full case; (c) FullBorn case; (e) Born-Born case. The second column of the figure present the evolution in log-scale of the minimized cost functional versus iteration steps which correspond to images plotted in the first column. 

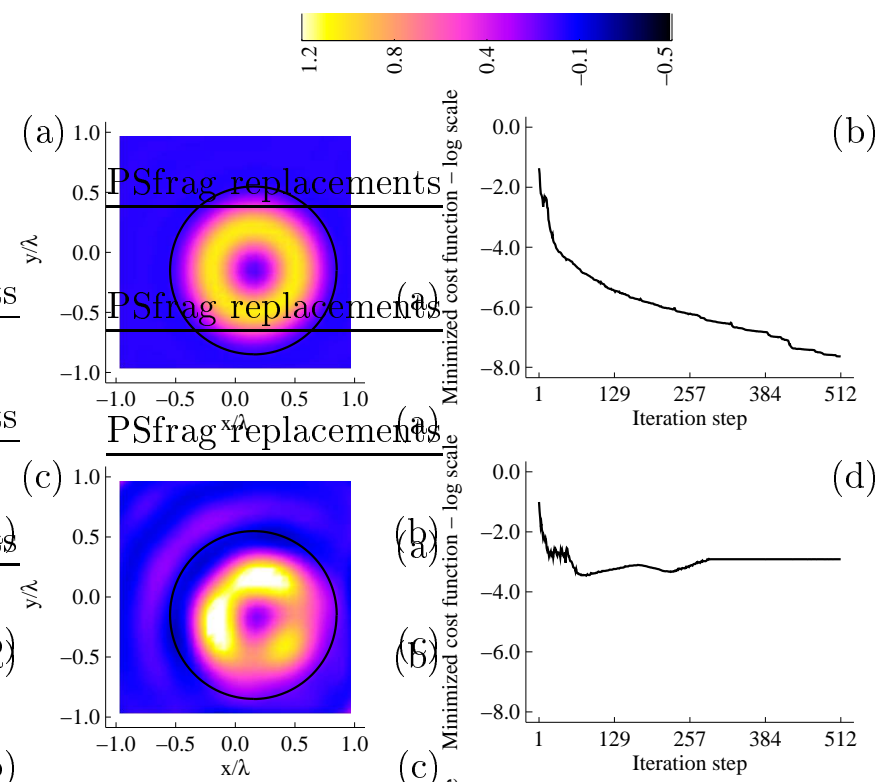

(b)
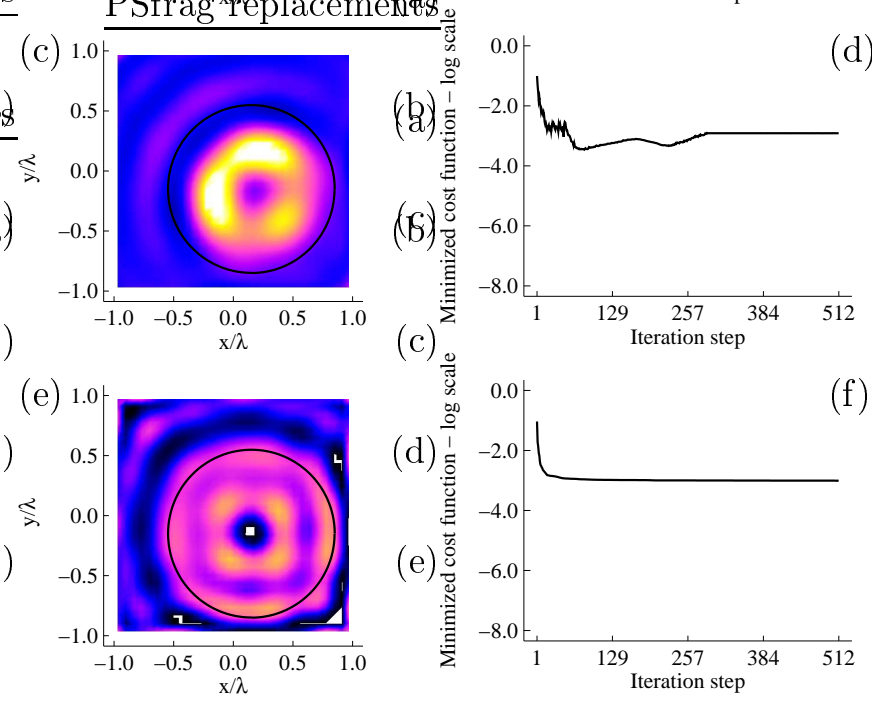

Fig. 11. Same as in Fig. 10 but with the object InHomoSin. 

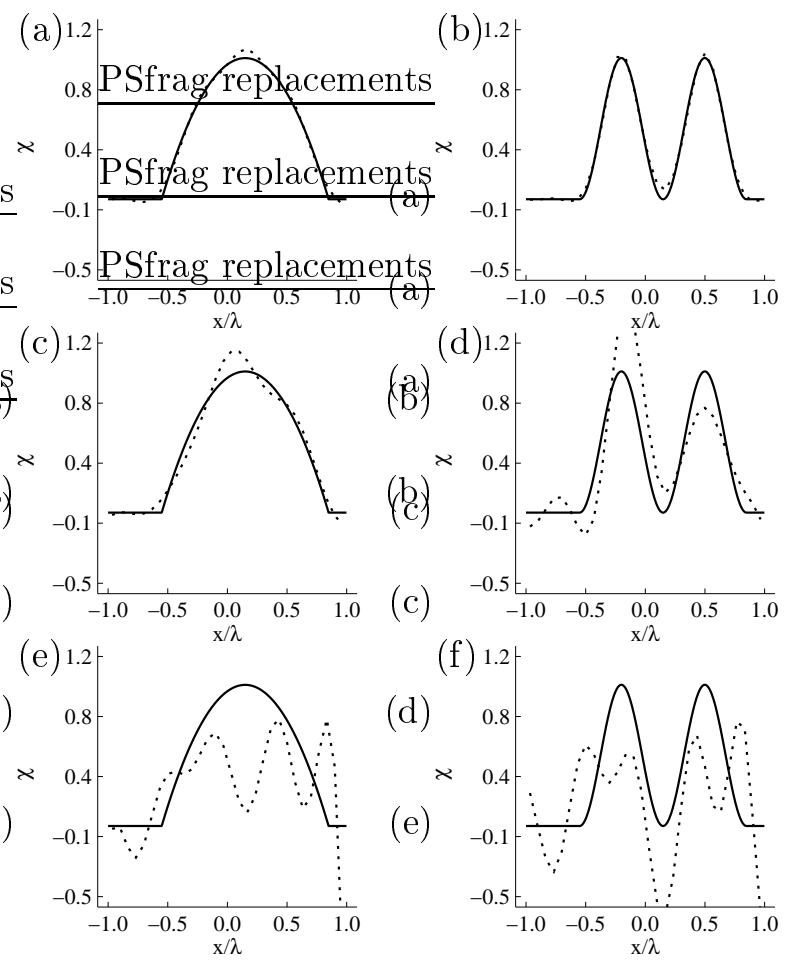

Fig. 12. Comparisons between the reconstructed profiles and the actual one along a horizontal line $y \approx-0.15 \lambda$ for the LunEBERG (first column) and InHOMOSin objects (second column). Full line stands for the actual profile while the dotted line corresponds to the reconstructed one. (a)(b) Full-Full; (c)(d) Full-Born and (e)(f) Born-Born. 

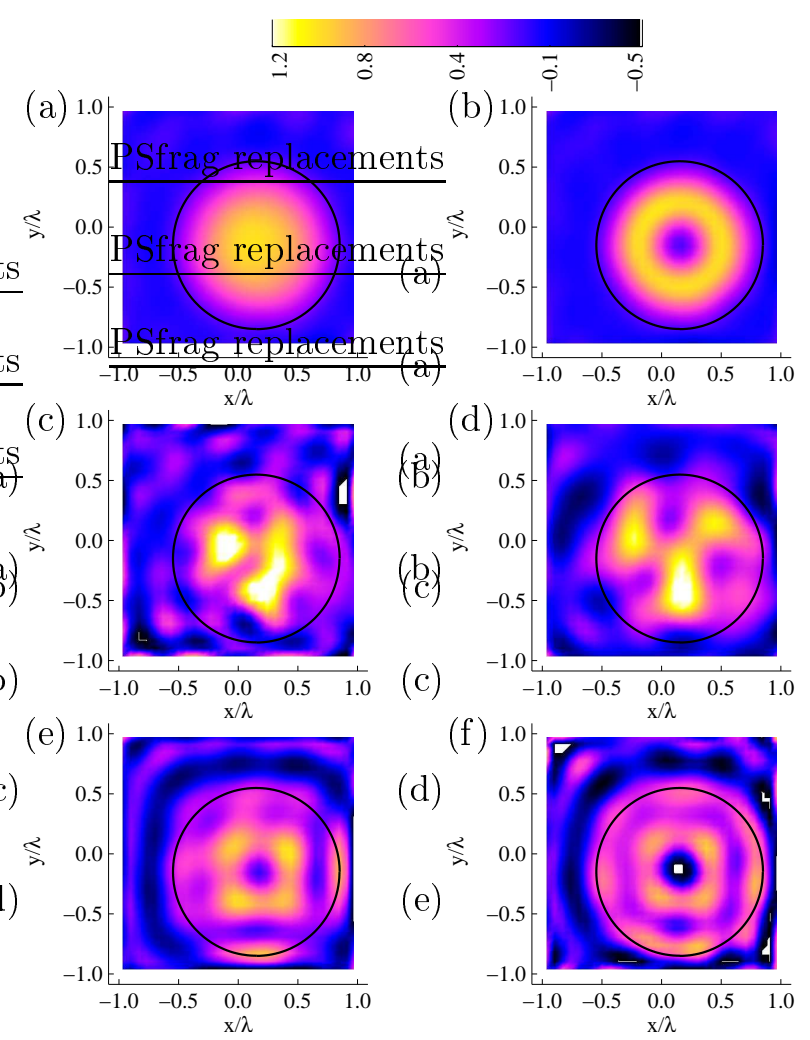

Fig. 13. Same as in Fig. 10 and Fig. 11 but with $10 \%$ additive noise in the data. First column corresponds to the LUNEBERG object while the second column corresponds to the INHOMOSIN object. 

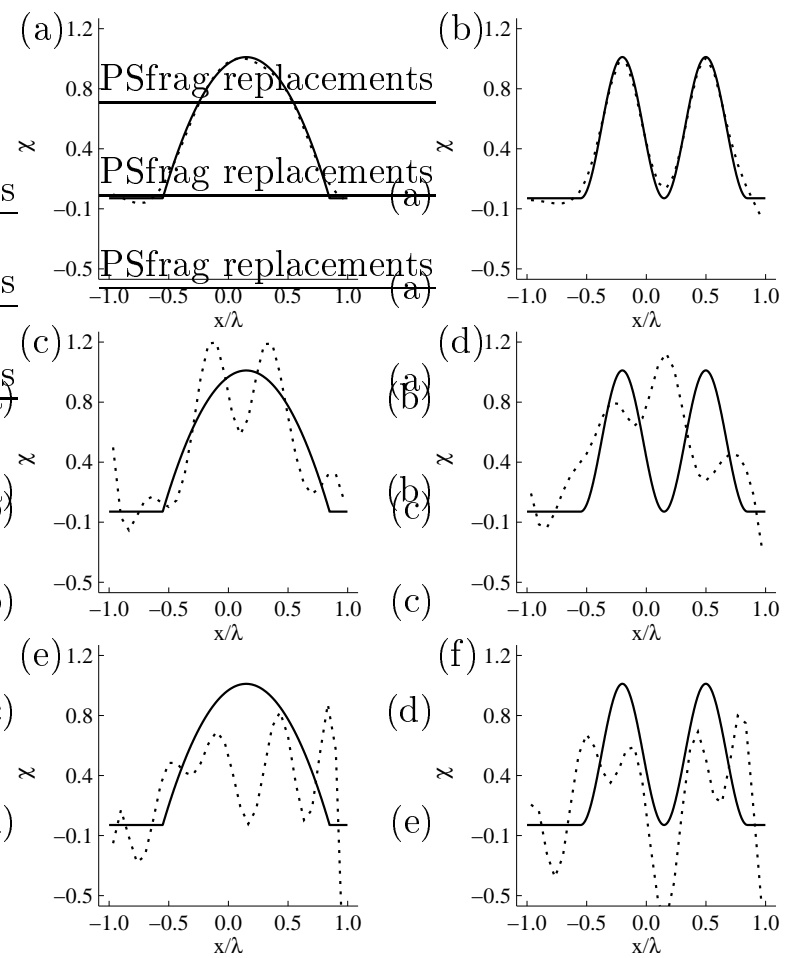

Fig. 14. Same as in Fig. 12 but with $10 \%$ additive noise. 